\title{
A noise SAR sidelobe suppression algorithm based on stable realization of apodization filtering
}

\author{
Xin Wu ${ }^{1,2 a)}$, Chang Liu ${ }^{1}$, and Yanfei Wang ${ }^{1}$ \\ ${ }^{1}$ Institute of Electronics, Chinese Academy of Sciences, Beijing, 100190, China \\ ${ }^{2}$ Graduate University of Chinese Academy of Sciences, Beijing, 100039, China \\ a)19861002wuxin@163.com
}

\begin{abstract}
Investigated here is a novel approach to the sidelobe suppression for noise synthetic aperture radar (SAR). The suggested strategy is a stable realization of apodization filtering (SRAF) with detailed implementation. Base on SRAF, a specific algorithm for noise SAR sidelobe suppression is proposed combining with range-Doppler (RD) algorithm. It applies SRAF to range correlation and azimuth compression to control sidelobes. The mathematic representations are presented and experimentation is performed. This algorithm proved that it can depress the sidelobes effectively and keep the energy of the mainlobe. Besides, it is not sensitive to noise disturbance and can avoid obvious SNR degradation.
\end{abstract}

Keywords: noise SAR, sidelobe suppression, SRAF, correlation Classification: Microwave and millimeter wave devices, circuits, and systems

\section{References}

[1] I. G. Cumming and F. H. Wong, Digital Processing of Synthetic Aperture Radar Data: Algorithms and Implementation, Artech House, Norwood, 2005.

[2] G. S. Liu, H. Gu, and W. M. Su, "The development of random signal radars," IEEE Trans. Aerosp. Electron. Syst., vol. 35, no. 3, pp. 770-776, July 1999.

[3] S. R. J. Axelsson, "Noise radar using random phase and frequency modulation," IEEE Trans. Geosci. Remote Sens., vol. 42, no. 11, pp. 23702384, Nov. 2004.

[4] D. S. Garmatyuk and R. M. Narayanan, "SAR imaging using fully random bandlimited signals," IEEE Antennas Propag. Society International Symposium., vol. 1-4, pp. 1948-1951, 2000.

[5] R. M. Narayanan, X. Xu, and J. A. Henning, "Radar penetration imaging using ultra-wideband (UWB) random noise waveforms," IEE Proceedings-Radar Sonar and Navigation., vol. 151, no. 3, pp. 143-148, June 2004.

[6] X. J. Xu and R. M. Narayanan, "Fopen sar imaging using uwb stepfrequency and random noise waveforms," IEEE Trans. Aerosp. Electron. Syst., vol. 37, no. 4, pp. 1287-1300, Oct. 2001. 
[7] H. Wang, R. M. Narayanan, and Z. O. Zhou, "Through-wall imaging of moving targets using UWB random noise radar," IEEE Antennas Wireless Propag. Lett., vol. 8, pp. 802-805, Aug. 2009.

[8] R. D. Fry and D. A. Gray, "CLEAN deconvolution for sidelobe suppression in random noise radar," Proc. IEEE 2008 Radar Conf., Adelaide, Australia, pp. 209-212, Sept. 2008.

[9] J. Cheng, B. C. Zhang, and Y. F. Wang, "Pulse Compression Methods for Random Noise Radar," Modern Radar., vol. 30, no. 2, pp. 40-43, Feb. 2008.

[10] C. Ni, Y. F. Wang, and X. H. Xu, "A SAR sidelobe suppression algorithm based on modified spatially variant apodization," Sci China Tech Sci., vol. 53, no. 9, pp. 2542-2551, Sept. 2010.

[11] X. Xu and R. M. Narayanan, "Range sidelobe suppression technique for coherent ultra wide-band random noise radar imaging," IEEE Trans. Antennas Propag., vol. 49, no. 12, pp. 1836-1842, Dec. 2001.

[12] M. Y. Zou, Deconvolution and signal recovery, National Defence Industry Press, Beijing, 2001.

\section{Introduction}

Synthetic aperture radar (SAR) is a kind of high resolution imaging radar in which backscattered microwave pulses are collected by an airplane or satellite [1]. Noise radar refers to radar whose transmitted signal is a microwave noise or is modulated by noise source. It performs pulse compression via direct correlation in time domain $[2,3]$. Noise SAR combines noise radar and SAR technique with both advantages of the two individuals, and it has become a useful sensor in many applications $[4,5,6,7]$.

The pulse responses of noise SAR along range and azimuth generally suffer from high sidelobes due to the finite two-dimensional frequency domain support of imaging system, therefore, sidelobe reduction is significant to noise SAR. Some sidelobe suppression methods have been proposed [8, 9, 10], however, they are not consistent with the direct range sampling in time domain of noise SAR. Apodization filtering is proposed to solve sidelobe problem of noise radar, and it has been proved feasible and effective [11], whereas many problems still to be resolved about it. For instance, realization process is not stable, restrictive conditions are excessive, etc.

In this study, a modified apodization filtering technique SRAF is proposed. The noticeable advantage of SRAF is stable realization. Moreover, it expands the traditional filter from using in range filtering to both in range and azimuth filtering. This paper is organized as: Section 2 introduces the proposed method SRAF. Section 3 presents the algorithm that applying SRAF to noise SAR sidelobe control. And experimental results are discussed in Section 4. Finally, conclusive remarks are provided in Section 5. 


\section{Principle and process of proposed algorithm}

\subsection{Principle of apodization filtering}

In Fourier analysis, the frequency domain data is multiplied by window functions to achieve great reduction of sidelobes. In terms of convolution theorem, multiplication in frequency domain is equivalent to convolution in time domain, so that a corresponding filter in time domain can be obtained. Apodization filtering technique is using time domain filtering to get high resolution output [11]. The principle can be expressed as

$$
\mathrm{G}(\tau)=\mathrm{A}(\tau) \otimes \mathrm{F}(\tau)
$$

where $\mathrm{A}(\tau)$ is original response with obvious sidelobes, $\mathrm{G}(\tau)$ is prospective response with great sidelobe suppression, $\mathrm{F}(\tau)$ is the goal filtering function, and $\otimes$ denotes convolution operation. The response generally is discrete, so Eq. (1) should be rewritten to matrix form that is shown as

$$
\mathrm{K} * \mathrm{~F}=\mathrm{G}
$$

where $\mathrm{K}$ is coefficient matrix, $\mathrm{F}$ and $\mathrm{G}$ are filtering vector and desired response vector that are the sampled versions of $\mathrm{F}(\tau)$ and $\mathrm{G}(\tau)$.

\subsection{Specific processing of SRAF}

From above, the objective is getting filtering vector $\mathrm{F}$ that can be denoted as

$$
\mathrm{F}=\left[\begin{array}{llll}
\mathrm{f}_{1} & \mathrm{f}_{2} & \cdots & \mathrm{f}_{\mathrm{n}}
\end{array}\right]_{\mathrm{n} \times 1}^{\mathrm{T}}
$$

where $\mathrm{n}$ is the filter length that should be determined in advance. Getting $\mathrm{F}$ is solving a first kind integral equation which generally represents ill-posed system, so that the stability of the adopted method is as important as its efficiency. The proposed method is introduced with great stability, and it contains three important steps that getting coefficient matrix, getting desired response vector and resolving equation to find filtering vector.

Coefficient matrix $\mathrm{K}$ and desired response vector $\mathrm{G}$ should be obtained firstly. The digitized form of original response $\mathrm{A}(\tau)$ can be expressed as

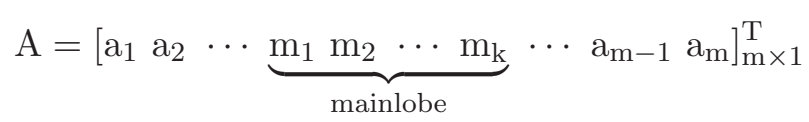

where $\mathrm{m}_{\mathrm{i}}(\mathrm{i}=1,2 \cdots \mathrm{k})$ are elements of mainlobe, $\mathrm{m}$ is the dimension of $\mathrm{A}$. The matrix K can be constructed by Eq. (5)

$$
\mathrm{K}=\left[\begin{array}{cccc}
\mathrm{a}_{1} & & & \\
\mathrm{a}_{2} & \mathrm{a}_{1} & & \\
\vdots & \mathrm{a}_{2} & \ddots & \\
\mathrm{a}_{\mathrm{m}} & \vdots & & \mathrm{a}_{1} \\
& \mathrm{a}_{\mathrm{m}} & & \mathrm{a}_{2} \\
& & \ddots & \vdots \\
& & & \mathrm{a}_{\mathrm{m}}
\end{array}\right]_{(\mathrm{m}+\mathrm{n}-1) \times \mathrm{n}}
$$


The desired response $\mathrm{G}$ is selected as follows: set the amplitude of all the sidelobe points to be zero, and set the amplitude of the mainlobe points to be same as original response A. It can be determined as

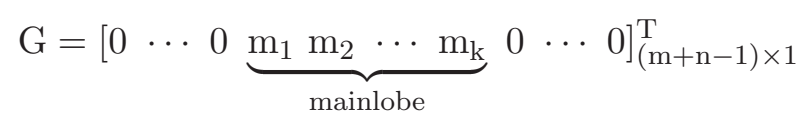

where $m_{i}(i=1,2 \cdots k)$ are located at the center of $G$.

After getting the $\mathrm{K}$ and $\mathrm{G}$, a suitable approach to finding the filtering vector should be applied. Considering our problem, a sequence with finite length should be utilized to approach the true solution which makes plentiful nonzero elements of original response become zero. Thus the solution must have several discontinuous points. Total variation (TV) method is introduced to solve our problem with advantage of non-restricting solution to be smooth [12]. It is able to retain the discontinuous boundary of the solution. The equation as Eq. (7) with initial guess solution $\mathrm{F}^{0}=[0, \cdots, 0]_{\mathrm{n} \times 1}^{\mathrm{T}}$ should be iteratively resolve.

$$
\left[\mathrm{K}^{\mathrm{T}} \mathrm{K}+\alpha \tilde{\mathrm{D}}^{\mathrm{T}} \mathrm{Q}_{\mathrm{m}}^{-1} \tilde{\mathrm{D}}\right] \mathrm{F}^{\mathrm{m}+1}=\mathrm{K}^{\mathrm{T}} \mathrm{G}
$$

where $\alpha>0$ is a regularization parameter determined by L-curve, $\mathrm{Q}$ is a diagonal matrix, the subscript of $\mathrm{Q}$ and superscript of $\mathrm{F}$ denote iterative steps. The matrices of $\tilde{D}$ and $\mathrm{Q}_{\mathrm{m}}$ can be obtained by Eq. (8) and Eq. (9).

$$
\begin{gathered}
\tilde{\mathrm{D}}=\left[\begin{array}{ccccc}
-\mathrm{a} & & & & \\
\mathrm{b} & -\mathrm{a} & & & \\
& \mathrm{b} & \ddots & & \\
& & \ddots & \ddots & \\
& & & \mathrm{b} & -\mathrm{a}
\end{array}\right]_{\mathrm{n} \times \mathrm{n}} \\
\mathrm{Q}_{\mathrm{m}}(\mathrm{k}, \mathrm{k})=\sqrt{\left[\mathrm{F}^{\mathrm{m}}(\mathrm{k})-\mathrm{F}^{\mathrm{m}}(\mathrm{k}-1)\right]^{2}+\beta^{2}}
\end{gathered}
$$

where $\mathrm{a}$ and $\mathrm{b}$ are 0.665 and $0.1755[12], \mathrm{k}=2,3, \cdots, \mathrm{n}$ represents the kth element of $\mathrm{F}^{\mathrm{m}}, \beta$ is a non-negative parameter that can be regulated. Besides, $\mathrm{Q}_{\mathrm{m}}(1,1)=\sqrt{\left[\mathrm{F}^{\mathrm{m}}(1)\right]^{2}+\beta^{2}}$. Through iteratively solving Eq. (7), we can successively obtain $\mathrm{F}^{1}, \mathrm{~F}^{2}, \mathrm{~F}^{3}, \cdots$ until $\mathrm{F}^{\mathrm{n}}$ which is convergent to threshold. Let $\mathrm{F}=\mathrm{F}^{\mathrm{n}}$ as the filtering vector, the result of multiplying coefficient matrix $\mathrm{K}$ with filtering vector $\mathrm{F}$ is the filtered output with great suppression of sidelobes. The flowchart of SRAF is shown as Fig. 1.

\section{Sidelobe suppression for noise SAR based on SRAF}

The noise SAR system is demonstrated as Fig. 2 a). The range pulse compression performance is weakened if the emitted signal passes through a band-pass filter before transmission. The experiments show that the peak sidelobe level ratio (PSLR) and integrated sidelobe level ratio (ISLR) of correlation outputs fluctuate at $-13.6 \mathrm{~dB}$ and $-9.8 \mathrm{~dB}$. The pulse response along azimuth is sinc function with high sidelobes level. Therefore, sidelobe reduction both of 


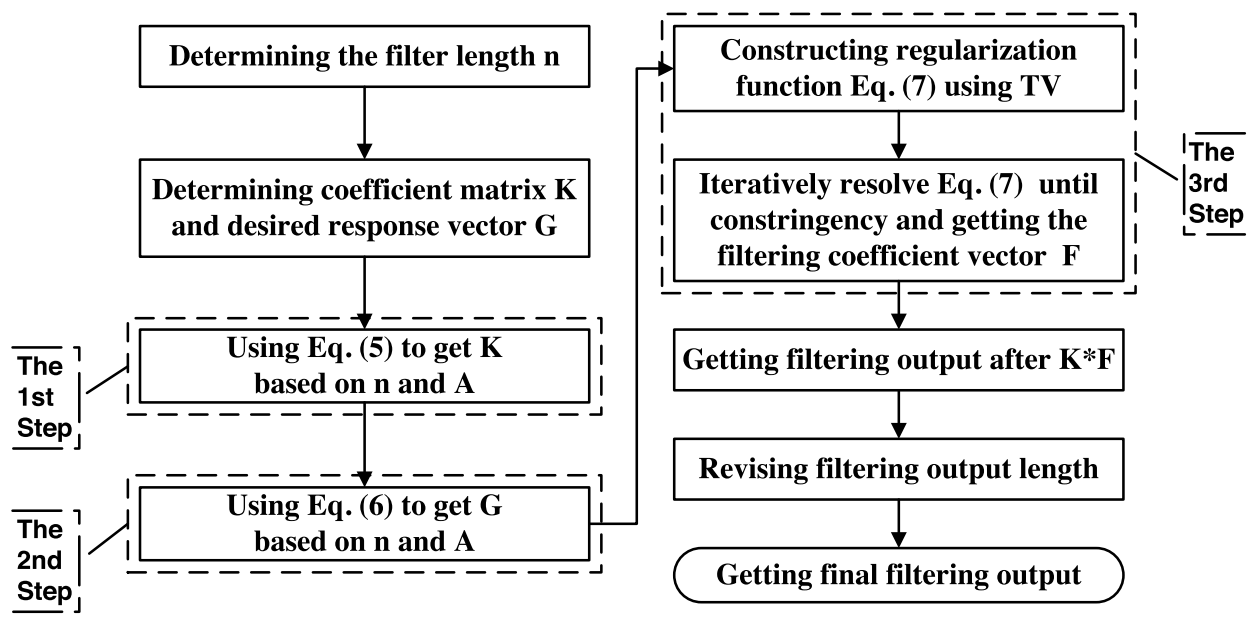

Fig. 1. Flowchart of SRAF

Transmission Signal

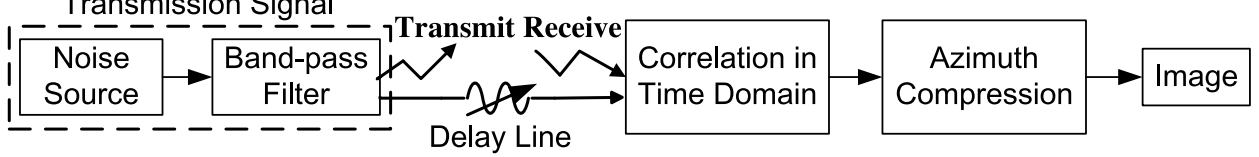

a) General system of noise SAR

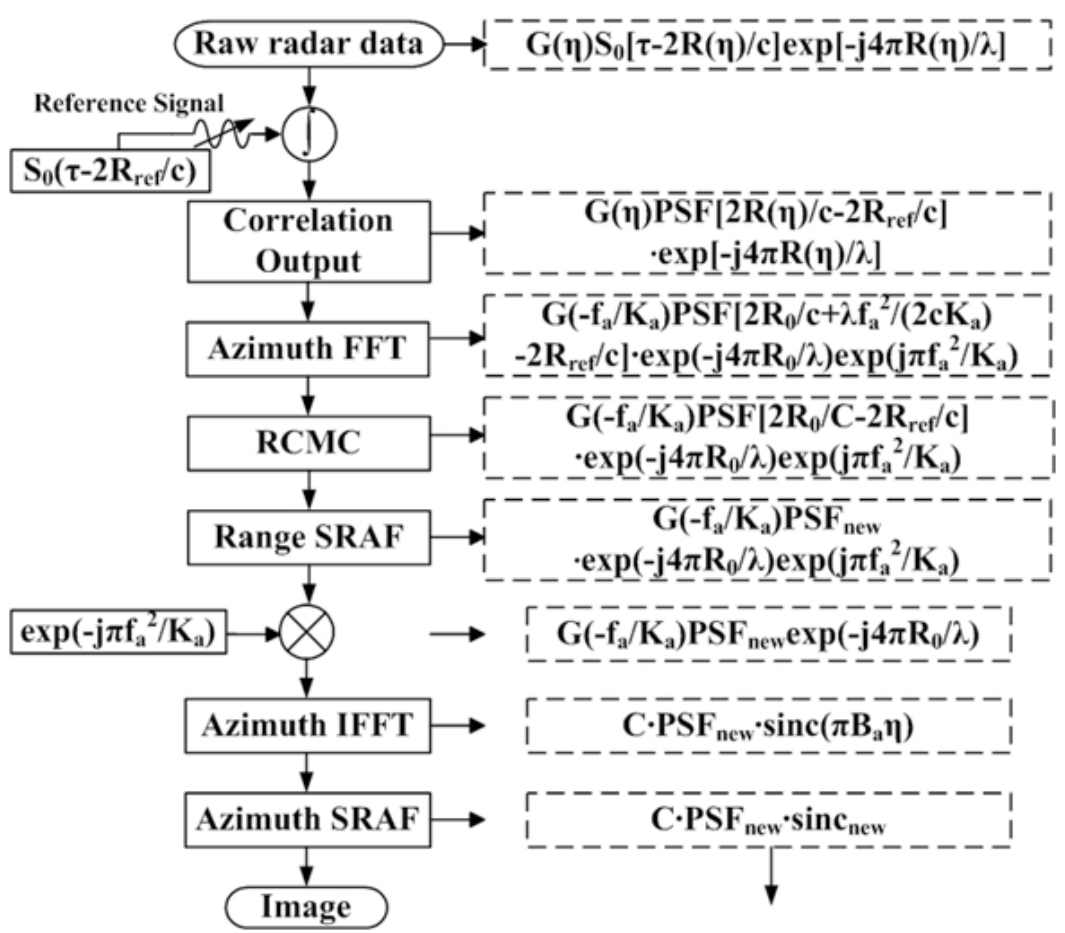

b) Flowchart of SRAF-RD

Fig. 2. Noise SAR system and flowchart of SRAF-RD

range and azimuth response is of particular importance. SRAF can be used in range correlation and azimuth compression to suppress sidelobes for noise SAR imaging process. Here, SRAF is combined with range-Doppler (RD) algorithm, which is short for SRAF-RD, as presented in Fig. $2 \mathrm{~b}$ ).

As can be seen from Fig. 2 b), left is the flowchart of SRAF-RD and right is the corresponding mathematic representations. The parameters in 
Fig. $2 \mathrm{~b}$ ) are illustrated as follows: $\mathrm{c}$ is the speed of light, $\lambda$ is the carrier wavelength, $\tau$ is the fast (range) time while $\eta$ is the slow (azimuth) time, $\mathrm{R}_{0}$ is the vertical distance between the target and the flight route, $\mathrm{R}_{\text {ref }}$ is reference range supplied by delay line, $\mathrm{f}_{\mathrm{a}}$ is azimuth frequency, $\mathrm{K}_{\mathrm{a}}$ is azimuthfrequency rate equaling to $2 \mathrm{v}^{2} /\left(\lambda \mathrm{R}_{0}\right)$ where $\mathrm{v}$ is the velocity of the sensor, $\mathrm{B}_{\mathrm{a}}$ is the bandwidth of azimuth, and $\mathrm{C}$ is a constant. The functions in Fig. $2 \mathrm{~b}$ ) are explained as: $\mathrm{S}_{0}(\tau)$ is the band-limited transmission signal, $\mathrm{R}(\eta)$ is the instantaneous range modeled as $\left[\mathrm{R}_{0}^{2}+(\mathrm{v} \eta)^{2}\right]^{1 / 2}, \mathrm{G}(\eta)$ is the weighting in azimuth from the antenna pattern, $\operatorname{PSF}(\cdot)$ and $\operatorname{sinc}(\cdot)$ are the results of range correlation and azimuth compression with high sidelobe level, while $\mathrm{PSF}_{\text {new }}$

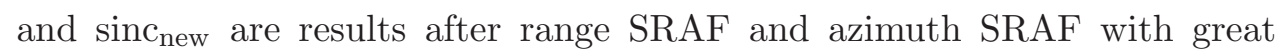
sidelobes reduction.

\section{Implementation and experimental results}

In this section, experimental results are provided to illustrate the effects of SRAF implemented on noise SAR imaging. The system parameters are shown as follows: carrier wavelength $\lambda=0.3 \mathrm{~m}$, transmission signal bandwidth $\mathrm{B}_{\mathrm{r}}=100 \mathrm{MHz}$, pulse duration $\mathrm{T}_{\mathrm{r}}=10 \mu \mathrm{s}$, sampling time interval $\Delta \tau=5 \mathrm{~ns}$, pulse repetition frequency $\mathrm{PRF}=400 \mathrm{~Hz}$, sensor velocity $\mathrm{v}=200 \mathrm{~m} / \mathrm{s}$, and slant range of scene center is $11.8 \mathrm{~km}$. The filtering length for the range and the azimuth directions are 2000 and 600 that approximate the range and the azimuth sample points counts.

Fig. 3 demonstrates the imaging results before and after SRAF process. Fig. $3 \mathrm{a}$ ) and Fig. $3 \mathrm{~b}$ ) are the profiles along the range direction and the azimuth direction of noise SAR image, where solid line are the results filtered by SRAF, and broken line are the original outputs without process. From Fig. 3 a) and Fig. 3 b), we can deduce that after SRAF filtering, the PSLR and ISLR of range output decrease from $-13.56 \mathrm{~dB}$ and $-9.73 \mathrm{~dB}$ to $-35.98 \mathrm{~dB}$ and $-21.66 \mathrm{~dB}$, respectively, while the corresponding values of azimuth output vary from $-13.44 \mathrm{~dB}$ and $-10.52 \mathrm{~dB}$ to less than $-60 \mathrm{~dB}$. Besides, the mainlobe is not broadened whose energy is kept well. Fig. 3c) and Fig. 3d) show the imaging results of a point target without any disposal and weighted by SRAF. Compared the two figures, there is a great suppression both of the range sidelobes and the azimuth sidelobes after SRAF. The presence of noise disturbance is also considered to test the anti-disturbance performance of SRAF and the SNR degradation. The results are illustrated as Fig. 3e) and Fig. 3 f). As the two figures shown, they are the range profiles and the azimuth profiles of the outputs without and after SRAF when SNR $=10 \mathrm{~dB}$ and SNR $=20 \mathrm{~dB}$. After SRAF filtering, the power of useful information is kept well while the noise is suppressed effectively, so the outputs don't appear obvious SNR degradation. SRAF is not sensitive to the SNR of the original signature, because the range correlation efficiently depresses the influence of noise due to the non-correlation between the transmission signal and noise disturbance. 


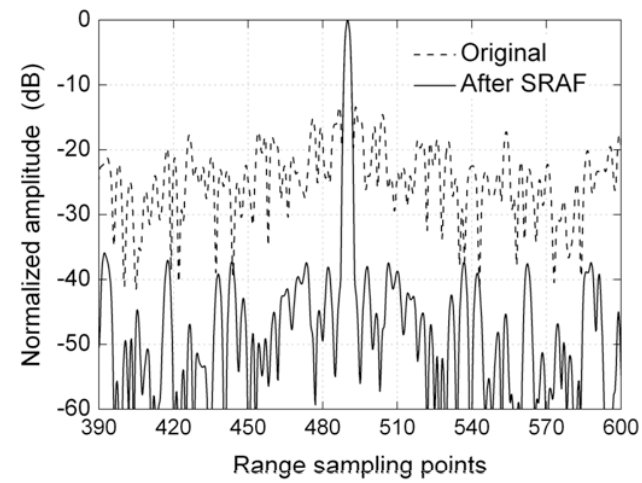

a) Profile of range

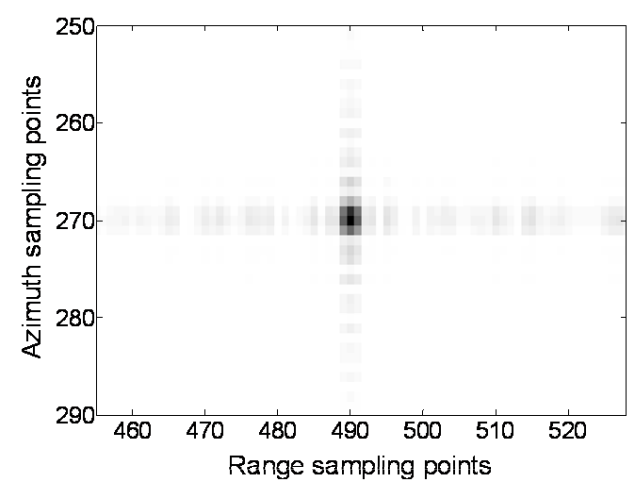

c) Original output

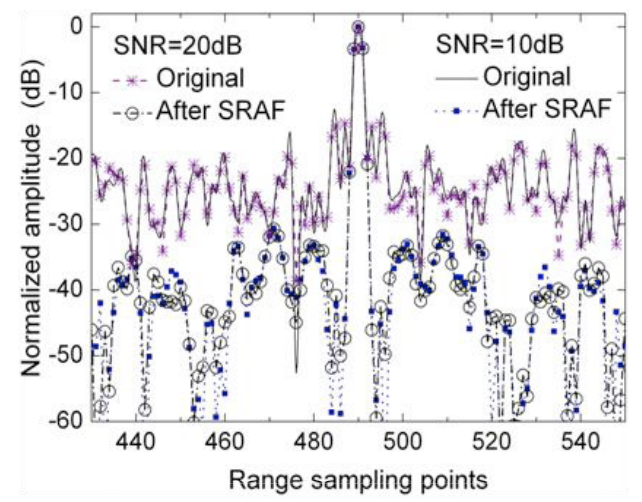

e) Profile of range with noise

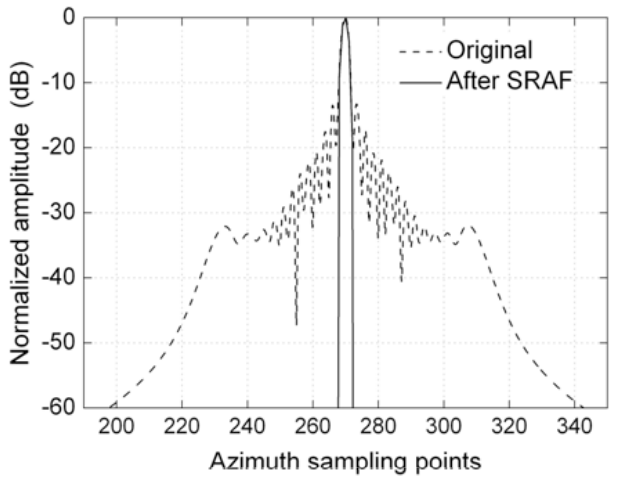

b) Profile of azimuth

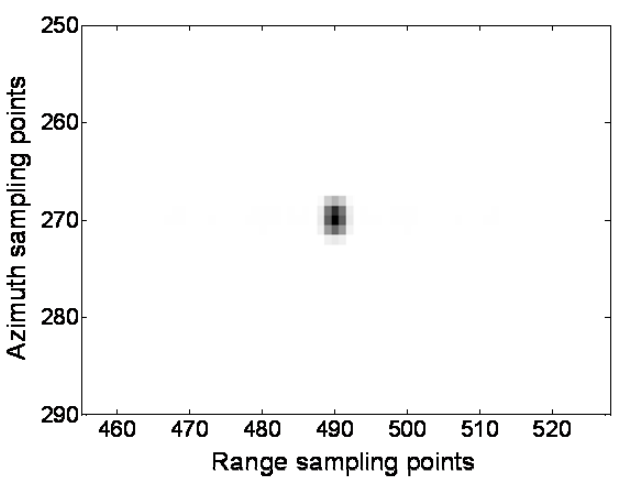

d) Output after SRAF

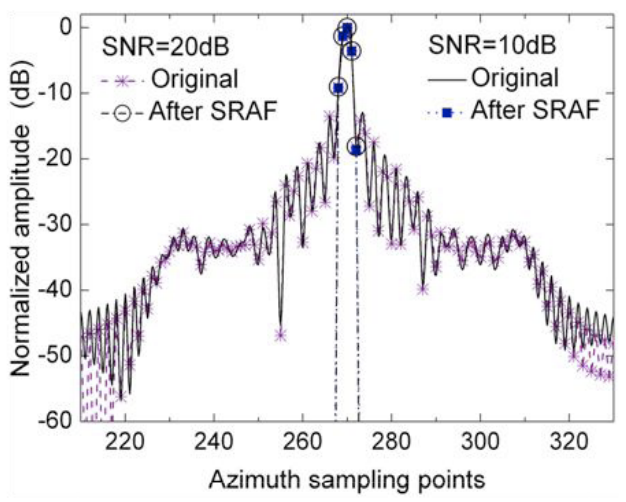

f) Profile of azimuth with noise

Fig. 3. Performance comparison before and after SRAF

\section{Conclusion}

In this work, a novel sidelobe suppression algorithm SRAF is applied to noise SAR imaging. This is a stable realization strategy for apodization filtering with three steps: getting coefficient matrix, getting desired response vector and using TV in solving equation to find filter vector. The pulse responses of noise SAR along the range and azimuth have high sidelobes levels in respect that the two-dimensional frequency domain support is finite. We combine SRAF with RD algorithm to control noise SAR sidelobes. The experimental results show that this algorithm is efficient in both range response and azimuth response. After SRAF process, sidelobe reduction of greater than $20 \mathrm{~dB}$ is possible and mainlobe energy is kept well. Moreover, the presented method is not sensitive to the SNR of the original signal and can avoid ob-

(c) IEICE 2011

DOI: 10.1587/elex.8.1769 Received August 24, 2011 Accepted October 12, 2011 Published November 10, 2011 
vious SNR degradation. Therefore, we can obtain high-quality noise SAR images using SRAF.

\section{Acknowledgments}

This work was supported by the Department of Airborne Microwave Remote Sensing System of Institute of Electronics. We appreciate the assistance of Research Fellow Gao Xin who provided many helpful comments. 